\title{
Prepubertal vaginal discharge: Vaginoscopy to rule out foreign body
}

\author{
Saniye Ekinci, İbrahim Karnak, Feridun Cahit Tanyel, Arbay Özden Çiftçi \\ Department of Pediatric Surgery, Hacettepe University Faculty of Medicine, Ankara, Turkey. \\ E-mail: sekinci@hacettepe.edu.tr \\ Received: 6 April 2015, Accepted: 27 April 2015
}

SUMMARY: Ekinci S, Karnak İ, Tanyel FC, Çiftçi AÖ. Prepubertal vaginal discharge: Vaginoscopy to rule out foreign body. Turk J Pediatr 2016; 58: 168-171.

This clinical study is designed to evaluate the results of vaginoscopies performed to rule out vaginal foreign body in prepubertal girls with vaginal discharge.

Medical records of all prepubertal patients who underwent vaginoscopy to rule out vaginal foreign body between 2004 and 2013 were reviewed retrospectively. All patients were evaluated by pediatricians prior to surgical consultation. Vaginoscopy is performed in the operating room under general anesthesia.

During the study period, 20 girls with persistent vaginal discharge with a mean age of 6.8 years (1-13 years) underwent vaginoscopy to rule out vaginal foreign body. Six patients had bloody vaginal discharge and 4 had recurrent vaginal bleeding lasting for more than one month. Ten patients had purulent vaginal discharge lasting for 1-7 months. None of vaginal cultures revealed pathological bacteria or candida species. Preoperative imaging techniques revealed vaginal foreign body in one patient only. Vaginoscopy demonstrated vaginal foreign bodies in four patients. Foreign bodies were grass inflorescence, safety pin and undefined brownish particles $(n=2)$, which may be pieces of toilet paper or feces. There was no complication related to vaginoscopy and removal of foreign body. Hymen integrity was preserved in all patients.

Persistent or recurrent vaginal discharge in prepubertal girls should raise the suspect of vaginal foreign body. Continuous flow vaginoscopy is mandatory to detect and remove any vaginal foreign body. Early diagnosis would prevent complications secondary to long-standing foreign bodies.

Key words: vaginal discharge, child, vaginoscopy.

Vaginal discharge is the most common gynecological complaint in prepubertal girls. Etiology of vaginal discharge may be nonspecific vulvovaginitis, infections, inflammatory or dermatologic conditions, precocious puberty, trauma, malignancy, sexual abuse or foreign body in vagina ${ }^{1-4}$. Undetected vaginal foreign bodies can cause important complications such as vaginal stenosis, vesicovaginal fistula, ureteral obstruction and renal failure ${ }^{5-8}$. Thus, it is important to rule out existence of a foreign body in a prepubertal girl with vaginal discharge. Pelvic X-ray, ultrasonography or magnetic resonance imaging can help the diagnosis but examination under anesthesia and vaginoscopy is mandatory for the exact diagnosis and removal of foreign body. Vaginoscopy allows thorough examination of vagina to detect any sign of abuse, infection or tumor, which, should be considered in differential diagnosis. During this examination any suspected lesion can be biopsied and foreign bodies can be removed with the help of foreign body forceps. Also, continuous flow of saline during the procedure irrigates the vaginal space and any tiny particle, which may be the cause of discharge, may be removed.

In this study the authors present the results of vaginoscopies performed to rule out vaginal foreign body in prepubertal girls with vaginal discharge. 


\section{Material and Methods}

Following the approval of Human Research Ethic Committee of Hacettepe University, medical records of all prepubertal patients who underwent vaginoscopy to rule out vaginal foreign body between 2004 and 2013 were reviewed retrospectively. Age, presenting symptoms, duration of symptoms, results of clinical, laboratory and radiological examinations prior to vaginoscopy and results of vaginoscopy are reviewed. All patients were evaluated by pediatricians prior to surgical consultation.

Vaginoscopy is performed in the operating room under general anesthesia. Careful external examination in dorsal lithotomy position with special care to detect any sign of sexual abuse was performed. Vaginal culture was obtained in the presence of a suspected infection. Vaginal canal was visualized by $10 \mathrm{Fr}$ pediatric cystoscope with continuous flow of normal saline. Vaginal distension was maintained by holding labia closely together to inspect vaginal canal and cervix. Any foreign body was removed with the help of a foreign body forceps or by irrigation of vaginal canal profusely.

\section{Results}

During the study period 20 girls with persistent vaginal discharge with a mean age of 6.8 years (1-13 years) were referred to our clinic to rule out vaginal foreign body. Six patients had bloody vaginal discharge and 4 had recurrent vaginal bleeding lasting for more than one month. Ten patients had purulent vaginal discharge lasting for 1-7 months.

Clinical examination and laboratory investigations did not indicate precocious puberty in any of children. Four children with recurrent vaginal bleeding were diagnosed as premature menarche after any other cause of vaginal bleeding including the vaginal foreign body was ruled out. One of the patients with the complaint of persistent vaginal bleeding was later diagnosed as Munchausen Syndrome by proxy and another had a bleeding disorder.

All patients with vaginal discharge had preoperative vaginal cultures, none of which showed any pathological bacteria or candida species. Twelve patients had 1-3 courses of antibiotic treatment prior to the vaginoscopy. Since the discharge was persistent they were referred to rule out vaginal foreign body.

Three patients with bloody discharge had fine papillary projections of cervix or vagina, which were biopsied. Pathological examination of specimens revealed normal cervical and vaginal tissue. Examination under anesthesia and vaginoscopy did not reveal any sign of sexual abuse in any of the patients.

Four patients had vaginal foreign bodies. Three of these patients had bloody discharge and 2 had purulent discharge. Foreign bodies were grass inflorescence, safety pin and undefined brownish particles $(n=2)$, which may be pieces of toilet paper or feces.

Clinical course was uneventful after vaginoscopy in all patients. There was no complication related to procedure and hymen integrity was preserved in all patients.

\section{Discussion}

A child with vaginal foreign body generally presents with vaginal discharge, which is the most common gynecologic symptom of prepubertal children. Vulvovaginitis is the most common cause of vaginal discharge in prepubertal children ${ }^{1-4}$. Simple measures such as wiping from front to back, omitting nylon or lycra underwear or any clothing that restricts air circulation to the genital area, showering instead of tub baths with shampoo or bubble baths will eliminate the discharge in most cases. However, further evaluation is necessary when discharge persists, contains blood or has foul smell. Vaginal cultures may demonstrate pathological bacteria or candida species. Sexual abuse, genital anomaly, and foreign bodies in vagina or malignancies such, as rhabdomyosarcoma and endodermal sinus tumor should also be considered.

None of our patients had positive cultures and all had normal pelvic ultrasonographic examinations. Pelvic X-ray demonstrated foreign body in one patient (Fig. 1). Imaging techniques may not be enough to demonstrate tiny or non-opaque vaginal foreign bodies in all cases ${ }^{9}$. Vaginoscopy is mandatory in the presence of any suspected vaginal foreign body.

Obtaining the history and the physical examination of a girl with vaginal discharge may be stressful and disturbing. Children mostly deny the insertion of any object. Gynecological 


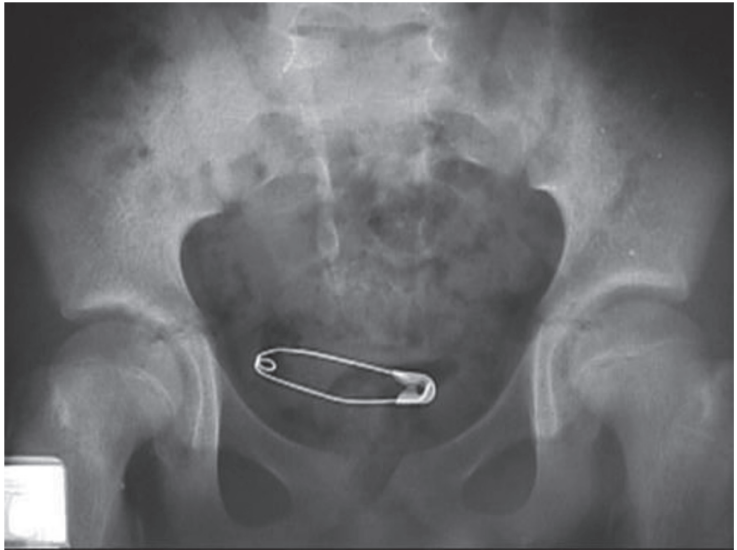

Fig 1. Pelvic X-ray demonstrating safety pin in vagina

examination in supine frog leg position may help the diagnosis but is not enough to detect intravaginal foreign body. Instrumentation of vagina even by a pediatric speculum may cause pain and injure the intact hymen. Small children cannot cooperate during such an intimate examination. Examination under anesthesia and vaginoscopy allows thorough examination of external genitalia and vagina to detect any sign of abuse, infection, tumor or foreign body. During this examination any suspected lesion can be biopsied and foreign bodies can be removed. All of our patients had an uneventful postoperative course. Integrity of hymen was preserved in all patients and none had a complication secondary to procedure.

In this series, $50 \%$ of children had persistent vaginal discharge without blood. Two of these patients had tiny brownish foreign bodies in vagina, which were evacuated by irrigation of vagina by normal saline during continuous flow vaginoscopy.

Blood in diapers or panties may be related to genital tissues as well as urethra or anus. If the blood is not related to anal tissues or urethra it may be related to bacterial vaginitis, lichen sclerosus, genital warts, vaginal tumors or foreign bodies. Precocious puberty or ovarian tumors may also lead vaginal bleeding ${ }^{10-12}$.

Four patients with vaginal bleeding were diagnosed as premature menarche. Premature menarche is a benign self-limiting condition. It is considered when vaginal bleeding is not accompanied by any other sign of precocious puberty in a prepubertal girl and thorough investigations including vaginoscopy show no other cause of vaginal bleeding ${ }^{13-14}$.

In this series, two patients with vaginal bleeding had vaginal foreign bodies (safety pin and grass inflorescence). These patients had an uneventful postoperative course and symptoms resolved after the removal of foreign bodies.

One of our patients complaining of vaginal bleeding was later diagnosed as Munchausen Syndrome by proxy. Some patients may need a detailed psychological evaluation to diagnose child abuse including sexual abuse or Munchausen Syndrome by proxy. Besides, blood in panties should raise the suspect of sexual abuse ${ }^{10-12}$.

Vaginal bleeding may be the first sign of a hematological disorder including malignancy ${ }^{15,16}$. One of our patients with persistent vaginal bleeding was diagnosed as bleeding diathesis later on.

Many unusual objects such as small toys, hairpins and disc batteries have been removed from the vagina of small girls. Children generally insert these objects as they explore their body. On the other hand, vaginal foreign bodies may indicate sexual assault. Child abuse should be considered in patients with unusual objects in vagina ${ }^{17}$.

Long retained foreign bodies may lead to complications such as: pelvic abscess, vesicovaginal fistula, rectovaginal fistula and vaginal stenosis. These complications cause unusual signs and symptoms such as: dysuria, pelvic/abdominal pain, bleeding during defecation and urinary incontinence ${ }^{6-9,18}$. Any persistent or recurrent genitourinary symptom in prepubertal girls should raise the suspicion of vaginal foreign body. Continuous flow vaginoscopy is a simple safe technique to detect and remove any vaginal foreign body. Early diagnosis would prevent the severe complications secondary to long-standing foreign bodies.

\section{REFERENCES}

1. Dei M, Di Maggio F, Di Paolo G, Bruni V. Vulvovaginitis in childhood. Best Pract Res Clin Obstet Gynaecol 2010; 24: 129-137.

2. Garden AS. Vulvovaginitis and other common childhood gynaecological conditions. Arch Dis Child Educ Pract Ed 2011; 96: 73-78.

3. Rome ES. Vulvovaginitis and other common vulvar disorders in children. Endocr Dev 2012; 22: 72-83. 
4. McGreal S, Wood P. Recurrent vaginal discharge in children. J Pediatr Adolesc Gynecol 2013; 26: 205-208.

5. Simon DA, Berry S, Brannian J, Hansen K. Recurrent, purulent vaginal discharge associated with longstanding presence of a foreign body and vaginal stenosis. J Pediatr Adolesc Gynecol 2003; 16: 361-363.

6. Siddiqui NY, Paraiso MF. Vesicovaginal fistula due to an unreported foreign body in an adolescent. J Pediatr Adolesc Gynecol 2007; 20: 253-255.

7. Heintz J, Chason J, Kramer A. Bilateral ureteral obstruction caused by vaginal foreign body: a case report. Can J Urol 2009; 16: 4870-4872.

8. Evans JM, South MM, Karram MM. Vesicovaginal fistula due to remote history of vaginal foreign body. Female Pelvic Med Reconstr Surg 2012; 18: 374-375.

9. Smith YR, Berman DR, Quint EH. Premenarchal vaginal discharge: findings of procedures to rule out foreign bodies. J Pediatr Adolesc Gynecol 2002; 15: 227-230.

10. Imai A, Horibe S, Tamaya T. Genital bleeding in premenarcheal children. Int J Gynaecol Obstet 1995; 49: $41-45$.
11. Merritt DF. Evaluation of vaginal bleeding in the preadolescent child. Semin Pediatr Surg 1998; 7: 35-42.

12. Perlman SE. Management quandary. Premenarchal vaginal bleeding. J Pediatr Adolesc Gynecol 2001; 14: 135-136.

13. Murram D, Dewhurst J, Grant DB. Premature menarche: a follow-up study. Arch Dis Child 1983; 58: 142-143.

14. Saggese G, Ghirri P, Del Vecchio A, Papini A, Pardi D. Gonadotropin pulsatile secretion in girls with premature menarche. Horm Res 1990; 33: 5-10.

15. Blaicher W, Jäger U. Abnormal vaginal bleeding as first symptom of acute leukemia. Arch Gynecol Obstet 2001; 265: 55-56.

16. Ellis $\mathrm{MH}$, Beyth Y. Abnormal vaginal bleeding in adolescence as the presenting symptom of a bleeding diathesis. J Pediatr Adolesc Gynecol 1999; 12: 127-131.

17. Herman-Giddens ME. Vaginal foreign bodies and child sexual abuse. Arch Pediatr Adolesc Med 1994; 148: 195-200.

18. Shiryazdi SM, Heiranizadeh N, Soltani HR. Rectorrhagia and vaginal discharge caused by a vaginal foreign body--a case report and review of literature. J Pediatr Adolesc Gynecol 2013; 26: 73-75. 